\title{
Perturber et déloger les méthodologies en enseignement des STEM: de l'ingénierie au bricolage théorique pour une justice éco-sociale
}

\author{
Marc Higgins • Maria F. G. Wallace • \\ Jesse Bazzul \\ Published online: 14 September 2018 \\ (C) Ontario Institute for Educational Studies (OISE) 2018
}

\section{Pourquoi il est important de perturber et de déloger les méthodologies en enseignement des STEM}

On l'a répété maintes fois pendant plusieurs décennies et dans le cadre de différents paradigmes: les pratiques usuelles en enseignement des sciences, des technologies, de l'ingénierie et des mathématiques (STEM) produisent et reproduisent certains systèmes de dominance, qu'il s'agisse de patriarchie, d'hétéronormativité, de suprématie blanche, d'eurocentrisme, de (néo-)colonialisme, de capacitisme, de classisme, d'inégalités en milieu de travail, d'anthropocentrisme et d'autres encore. Heureusement, nombreux sont ceux et celles qui travaillent de façon active et créative pour (r)ouvrir l'enseignement des STEM à la possibilité d'une justice éco-sociale à venir, grâce à une pluralité d'approches, d'orientations et de positions productives: anti-oppressives, antiracistes et racialisées critiques, décolonisatrices et dé/colonisantes, homos, autochtones, égalitaires sur le plan des sexes, postcoloniales, participatives et communautaires, fondées sur les lieux (et critiques), interespèces, et bien d'autres. De plus, il existe de nombreux exemples de positions fortement critiques, ou au contraire complices, pour perpétuer ou pour contrer la logique de l'exclusion. Ce faisant, cependant, elles ne sont souvent que des tentatives dépolitisées et athéoriques qui continuent, bien que de manières différentes, à considérer comme autres ceux qui avaient été exclus. En tant que lecteurs dans le domaine, nous soulignons le fait que les efforts qui sont faits sont souvent centrés sur des questions de curriculum et de pédagogie; cela est tout à fait normal, car ces questions sont au cœur de l'enseignement des STEM. La question de savoir comment l'acquisition graduelle des connaissances sur la nature, les nombres et les technologies est conceptualisée et mise en place est

\footnotetext{
M. Higgins $(\square)$

Université de l'Alberta, Edmonton, AB, Canada

e-mail:marc1@ualberta.ca

M. F. G. Wallace

Millsaps College, Jackson, MS, USA

e-mail: wallamg@millsaps.edu

J. Bazzul

Université de Régina, Regina, SK, Canada

e-mail: jesse.bazzul@uregina.ca
} 
fondamentale, d'une part en termes de parcours curriculaires et de voies pédagogiques susceptibles de favoriser de tels apprentissages, et d'autre part en termes de destinataires visés. Par exemple, comme le rappellent Megan Bang et Amanda Marin (2015), l'inclusion de perspectives autochtones dans le curriculum amène une problématique différentielle si on n'affronte pas aussi la question du cadre épistémologique, ontologique et axiologique, tenu pour acquis et naturalisé, dans lequel on veut intégrer ces perspectives. Comme le soulignent Bang and Marin (2015), si l'enseignement des sciences continue d'être centré sur des phénomènes "établis » et sur des perspectives « reçues » en relation avec ces phénomènes (p. 531), qui d'une part se fondent sur le colonialisme blanc et la récursivité du privilège blanc, et d'autre part rejettent, minimisent ou nient les modes autochtones de vie en harmonie avec la nature, de même que la présence et l'avenir autochtones, l'inclusion ne sera que symbolique et ne servira qu'à distraire de la tâche exigeante qui consiste essentiellement à reconceptualiser et à recentrer la question de la dominance. Encore une fois, il est important de comprendre comment les curriculums, la pédagogie et ses fondements sont conceptualisés.

De même, la méthodologie est également ${ }^{1}$ un lieu important où entrent en jeu les mouvements du pouvoir, reproduisant de façon différentielle les articulations de la dominance. Bien que celles-ci se manifestent de manières beaucoup plus subtiles, nous devons nous demander comment les différentes méthodologies que nous utilisons dans nos pratiques de recherche, en tant que chercheurs en enseignement des STEM, contribuent à maintenir et à privilégier les parcours prédominants dans le domaine. À cette fin, soulignant les façons dont les disciplines déterminent ce qui compte comme savoirs, et, plus précisément, comme processus de production des savoirs, Linda Tuhiwei Smith et collègues (2016) posent la question suivante: «Les méthodologies sont-elles simplement de nouvelles technologies d'assimilation culturelle? » (p. 133). Pour Smith et collègues (2016), analyser la méthodologie signifie affronter les référents culturels qui continuent de se cacher dans notre constellation méthodologique de concepts (par exemple, la voix, l'identité, les données, la réflexivité, etc.). L'idée de poursuivre des objectifs critiques tout en conservant des méthodologies " conventionnelles », dont l'aspect « tenu pour acquis » ne peut aucunement mettre en lumière les conventions qui les sous-tendent, envoie un message subtil mais insidieux: les perspectives alternatives doivent être validées dans le cadre des normes de la dominance pour pouvoir « compter ». ${ }^{2}$ Il est nécessaire de décentrer activement ces notions « reçues » et d'inventer de multiples nouvelles façons d'assembler la théorie, la pratique et l'éthique.

Cependant, la volonté de perturber et de déloger les méthodologies ne signifie pas strictement une volonté de pluralisme méthodologique, une manière de «perdre la voie - mais bien perdre la notion qu'une seule « voie » puisse être préfixée et privilégiée par l'article défini » (Gough 2006, p. 640 , c'est nous qui soulignons). C'est aussi une volonté de «perturber les points de vue hégémoniques et les façons dont ils sont liés au fait que certains sujets s'attribuent la dominance » (McKinley, 2001, p. 76). Nous ne croyons nullement qu'une refonte critique et créative de la méthodologie soit (totalement) la panacée pour enrayer ce poison. Cela dit, le travail qui consiste à tenter de perturber et de déloger la méthodologie a au moins un objectif important, celui d'oser risquer d'échouer de manière innovatrice.

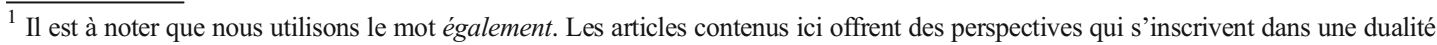
aussi/et plutôt que dans une opposition ou bien/ou: notre intention n'est pas de suggérer que l'attention sur la méthodologie devrait remplacer l'attention sur les curriculums et la pédagogie, mais bien s'y ajouter ou devenir un produit qui en est issu et le dépasse.

${ }^{2}$ Cependant, nous reconnaissons que le(s) domaine(s) de l'enseignement des STEM sont conservateurs aussi bien sur le plan politique que sur le plan théorique. Cela pose un défi particulier pour l'introduction de nouvelles formes de savoirs et de production des savoirs qui tiennent sérieusement compte des pratiques de pensée autres sans pour cela dépasser les normes de l'intelligibilité. Il s'agit peut-être là d'une demande, dans la lignée du chercheur cri Cash Ahenakew (2016), « d'écrire sur ce qui est invisible de façon à en faire remarquer l'absence, pour que cet invisible reste dans la mémoire et soit perçu comme un manque » (p. 333), une invitation à signaler nos complicités stratégiques.
} 


\section{De la conception de la recherche à la dé/conception de la recherche: de l’ingénierie au bricolage théorique}

Dans une étude encore pertinente aujourd'hui, Liz McKinley (2000) remet en question les façons dont la dominance a joué en enseignement des STEM, en soulignant comment la discipline réagit à la différence: « en masquant le pouvoir sous un voile d'innocence ». D'abord, McKinley (2000) met en lumière le fait que l'absence de savoirs (ou une position de «non-savoir ») sert souvent à (re)produire les normes du pouvoir; ensuite, dit-elle, " nous devons soulever ce voile d'innocence et nous interroger sur les façons dont les relations de domination et de subordination régissent les relations dans les salles de classe » (p. 76). Bien qu'elle parle ici de pédagogie et de curriculums, elle offre un prisme utile pour analyser les méthodologies de recherche en STEM.

Le panorama théorique des méthodologies de recherche en enseignement est en train de changer: la place occupée par une « innocence » qui serait issue d'un manque de savoirs n'est plus ce qu'elle était il y a vingt ans. ${ }^{3}$ Dans différents milieux d'enseignement, de nombreuses voix réclament qu'on s'engage de plus en plus dans des pratiques visant à perturber et à déloger les méthodologies: la dernière décennie en particulier se distingue dans ce sens (comme le montre par exemple la parution de numéros spéciaux de revues telles que Études culturelles $\leftrightarrow$ Méthodologies critiques, Études en enseignement et la Revue internationale des études qualitatives en enseignement). Il y a maintenant de multiples exemples productifs faisant une analyse critique des processus méthodologiques pour perturber et déloger les normes restrictives qui persistent sous la surface dans la recherche en éducation et dans ses concepts qui, si on ne les contre pas, (ré)articulent des formes de pouvoir oppressif. La place réservée à une " innocence " servant à masquer le pouvoir méthodologique n'a peut-être plus de raisons plausibles de ne pas s'attaquer aux référents tenus pour acquis dans des systèmes qui (re)produisent la dominance et les inégalités, et n'accordent aucune place à la responsabilité réciproque de part et d'autre des différences et du pouvoir.

Puisque les méthodologies (même celles qui sont critiques) partent souvent elles-mêmes des articulations majoritaires que nous voulons contrer en enseignement des STEM, nous arguons que la recherche peut et doit adopter une approche différente dès le départ, au stade de la conception du projet. Ailleurs (Higgins, Madden, Berard, Lenz-Kothe et Nordstrom, 2017), la notion de dé/conception est proposée pour se démarquer de ce que la conception de la recherche en vient à signifier: comme préexistant, comme séparé ou séparable des autres aspects de la recherche, voire comme moyen d'atteindre et de justifier les résultats. La conception de la recherche en enseignement des STEM ne doit pas, ou ne doit plus, se limiter à choisir, dans l'étalage méthodologique, la méthodologie la mieux ajustée aux besoins ou encore à la modifier ad hoc pour l'adapter. En d'autres termes, il faut résister à ce que Gayatri Spivak appelle la " rage de l'unité » (Spivak 1976, p. xvi), qui masque les différentes façons dont toutes les méthodologies finissent par constituer des ensembles vaguement définis de signifiés partiels, mis en situation et mis en relation entre eux. La dé/conception est « un moyen de travailler dans le cadre de la recherche, mais contre et au-delà de son façonnement ad hoc » (Higgins et al. 2017, p. 36). De plus, la dé/conception consiste d'une part à rendre compte, et assumer la responsabilité, de ce que les méthodologies conventionnelles signifient déjà, et d'autre part à participer au travail critique et créatif de redéfinir les signifiés des méthodologies. Voilà pourquoi, dans notre appel d'articles, nous avons posé la question suivante: Qu'est-ce que cela signifierait si on entreprenait de redéfinir la recherche de façon à perturber sérieusement, jusqu'à les déloger, les méthodologies en enseignement des sciences, des mathématiques, de l'ingénierie et des technologies (STEM) pour promouvoir la justice éco-sociale?

$\mathrm{Si}$ on analyse plus en détails la relation entre la conception et la dé/conception de la recherche, nous encadrons l'exploration de la question dans les métaphores dichotomiques et pertinentes de l'ingénierie et du bricolage: en suivant précisément la lecture (erronée) que fait Jacques Derrida (1976) de La pensée

\footnotetext{
${ }^{3}$ On trouve aussi des positions activement ancrées dans une attitude de «non-savoir » ou de « refus de savoir » qui persistent, mais la tâche de les explorer dépasse largement les limites d'un éditorial.
} 
sauvage de Claude Lévi-Strauss, dans laquelle il sépare, en même temps qu'il en brouille les distinctions, la métaphoricité des deux pratiques. La conception de la recherche agit souvent de manière prescriptive ou prohibitive, comme signifiant susceptible de suturer les processus signifiés de conception et de réalisation de la recherche. De la même façon, l'ingénierie est le mouvement qui va des fins vers les moyens, où l'ingénieur fait une sélection appropriée d'éléments dans " les discours de la logique formelle, et les sciences pures » (Spivak 1976, p. xix), en choisissant les concepts, les catégories et les construits déjà désignés pour leur processus (par exemple la recherche comme « meilleur ajustement» ou comme « meilleures pratiques »). Par conséquent, la « conception » de la recherche est souvent employée et comprise comme une méthode qui existe a priori, comme un ensemble d'instruments qui soutiennent la recherche (au lieu de la produire), comme un cadre qui inclut et englobe tous les éléments de son appareil conceptuel, qu'il s'agisse d'objectivité, de neutralité culturelle, de validité ou autre. Puisque les construits et les catégories mentionnés plus haut (re)produisent souvent implicitement les systèmes de pouvoir, ce numéro spécial est un appel au bricolage, plutôt qu'à l'ingénierie, de la recherche et des pratiques éducatives en enseignement des STEM.

Contrairement à l'ingénieur, « le bricoleur se contente de choses qui ont peut-être été conçues à d'autres fins » (Spivak 1976, p. xix). Le bricolage inverse la hiérarchie fins/moyens en privilégiant les moyens aux dépens des fins, ou le processus aux dépens du produit, même si cela implique un risque de ne pas atteindre les objectifs spécifiés. Comme le soutient Derrida (1976), les fins (par exemple le savoir, la vérité) et les moyens de produire les savoirs (c'est-à-dire la méthodologie) ne coïncident pour ainsi dire jamais. L'objectif d'atteindre la connaissance (complète) est non seulement empiriquement impossible, comme le pose LéviStrauss, il est aussi théoriquement impossible. Donc, pour Derrida (1976), « l'ingénieur doit toujours être aussi une sorte de bricoleur » (p. 139), mais il reconnaît également les limites mêmes du bricolage:

La seule faiblesse du bricolage - mais, vue comme faiblesse n'est-elle pas irrémédiable? - est sa totale incapacité de se justifier dans son propre discours. L'aspect « déjà présent » des instruments et des concepts ne peut être ni défait, ni réinventé. (pp. 138-139)

Tout comme les objectifs de l'ingénierie ne se réalisent jamais, Derrida met en garde contre la tentation de traiter les outils du bricoleur comme si eux-mêmes avaient toujours été présents. Plutôt, il invite à considérer les outils eux-mêmes comme les réalisations productives de bricolages passés et à venir.

Il y a toujours un besoin « de brouiller et d'utiliser simultanément les concepts [ainsi que les limites conceptuelles] dont nous pensons ne pas pouvoir nous passer pour penser ..., et que nous conservons aussi bien comme limite que comme ressource " (Lather 2007, p. 167-168). Enfin, le fait que le binôme ingénierie/bricolage est déjà poreux en soi, incite à une considération critique du bricolage et de ses fins, ou de ce qu'il produit: « tous les bricolages n'ont pas nécessairement la même valeur. Le bricolage fait sa propre autocritique » (Derrida 1976, p. 139). Toutefois, puisque la « trame méthodologique est, elle aussi, un produit fabriqué - une mise en œuvre performative et non séparable de la place où se conjuguent la théorie, la pratique et l'éthique » (Higgins et al. 2017, p. 17) - la conception méthodologique reste toujours ouverte à la déconstruction, à la politique et à la re(con)figuration.

Ce numéro spécial recueille les travaux de chercheurs en enseignement des STEM qui ont commencé le travail de bricoler la théorie, ${ }^{4}$ en se servant des théories interrogatives à l'intention des STEM, ou encore de théories destinées au départ à d'autres contextes, pour agir dans le cadre de la recherche sur l'enseignement des STEM afin de favoriser la justice sociale et écologique. Les manuscrits qui figurent ici sont vécus, participatifs et riches sur le plan critique, car ils bricolent à l'intérieur, à l'encontre et au-delà de ces espaces disciplinaires à tous les stades de la recherche, et comme pratique en enseignement: la conception, la livraison, l'analyse et la diffusion.

\footnotetext{
${ }^{4}$ Il est à noter que la formulation Bricolage de la théorie est un clin d'œil assez clair au Thinking with Theory, de Jackson et Mazzei (2012, 2017). 


\section{Le bricolage théorique: aperçu des articles recueillis}

Les articles contenus dans ce numéro sont partagés en deux volets. D'abord figurent les études qui bricolent $a ̀$ l'intérieur et à l'encontre des méthodologies de la recherche en enseignement des STEM, au moyen d'outils et de techniques destinées à être utilisées dans le cadre des STEM, mais refusent de s'en servir de la manière prévue. Ensuite viennent les études qui œuvrent au sein et au-delà des méthodologies de recherche en STEM en se servant d'outils et de techniques qui n'étaient pas destinées à l'enseignement des STEM. Ces catégories ne sont pas nécessairement mutuellement exclusives, car le matériel, les techniques et les concepts conçus à l'intention des STEM dépendent largement du positionnement, du lieu sociohistorique et du contexte.

Utilisation d'outils et de techniques conçues à l'intention de l'enseignement des STEM, mais refus de cette intention

Pour Sara Giordano, le bricolage est maintenant trop associé à des désirs masculinistes dans certains aspects de l'enseignement de la biologie synthétique. En suivant les théories de la spécialiste du féminisme décolonial Maria Lugones et de la chercheure en sciences sociales féministes Deboleena Roy à la fois pour utiliser et pour perturber le bricolage, Giordano participe à la redéfinition des signifiés que favorise le bricolage: vers des pratiques éducatives plus justes (plutôt que simplement plus nombreuses). Nikki Rotas, pour sa part, « applique » (Lather 2007) la notion baradienne de la diffraction dans son travail avec des enfants de 10 à 12 ans, dans le cadre d'un club parascolaire de robotique. Son but est de favoriser la compréhension d'un design capable de résister ou d'échapper aux parcours disciplinaires rigides issus de l'expérience et du design grâce à la personnification de cyborgs assortis de technologies portables. L'apprentissage peut-il mener à des épistémologies et ontologies alternatives émergeant de la participation à de multiples assemblages et appareils? C'est plus ou moins ce que soulignent Maria Wallace, Marc Higgins et Jesse Bazzul dans leur tentative de déloger le concept majoritaire de « nature » par le biais d'une petite enquête traçant les contours de la Nature, en respectant son potentiel inhérent, de plus en plus local, différentiateur et bizarre. Si l'enseignement est destiné à participer à la recherche de nouvelles formes d'existence collective, il devra explorer des conceptions de la nature qui sont plus relationnelles et contingentes, des conceptions visant à respecter et à encourager la différence. Dans un parcours similaire mais différent, Liz Defreitas et Nathalie Sinclair bricolent dans le cadre de l'espace mathématique très subtil de la probabilité, qui, avec d'autres, constitue la psychologie cognitive. En redéfinissant les signifiés de la relation entre la probabilité conventionnelle « ou bien/ou » et les apprenants incapables de percevoir et de formaliser les abstractions mathématiques, Defreitas et Sinclair jouent avec la probabilité quantique « aussi bien/et 》 pour inciter les chercheurs à considérer la superposition et l'indétermination dans les façons de concevoir les nombres (par exemple les formes) comme un cadeau différentiel potentiel.

Utilisation d'outils et de techniques non destinés à l'enseignement des STEM

Ce numéro spécial introduit également des outils, des techniques, des concepts et des méthodologies qui ne sont pas typiquement utilisées dans la recherche sur l'enseignement des STEM. La méthode cartographique de Michelle Wooten introduit, entre autres choses, un paysage ontologique dans la discipline de l'enseignement des sciences, de sorte que puissent se créer de nouvelles relations et de nouveaux niveaux d'intensité (au lieu d'être rejetés comme ils le sont souvent par l'enseignement traditionnel des sciences). Le travail de Wooten démontre, bien que de façon subtile, qu'il y a une grande insatisfaction devant le manque de cohérence qui existe entre des enseignants des sciences qui poursuivent des recherches déjà intégrées dans des cadres de recherche établis, et ceux qui aimeraient s'aventurer dans des parcours différents; par conséquent, la cartographie fournit un outil permettant d'établir des liens entre (tous) les aspects de ce travail. Dans une approche différente à la représentation, Shakhnoza Kayumova, Wenbo Zhang et Kate Scantlebury jouent avec des juxtapositions graphiques de vignettes image-texte pour amener à de nouvelles relations avec les données, et continuent dans la lignée de l'exploration ontologique pour trouver une 
pluralité de manières de « se lier » aux données et à l'analyse, et de personnifier nos relations avec elles. Les auteur(e)s, qui visent à remettre sérieusement en question l'idée que des méthodes de recherche n'ayant fait l'objet d'aucune analyse puissent maintenir en place des hiérarchies et des rapports de pouvoir hégémonique, se penchent sur les éventuels signifiés de cette remise en question pour ceux qui souhaitent favoriser l'équité en enseignement des sciences. Enfin, l'essai critique de Stacia Cedillo présente les façons dont les analyses de la BlackCrit constituent des approches utiles pour illustrer comment le mouvement STEM corporatif américain, et les sciences occidentales modernes en général, sont truffées de construits, de motivations et de traditions institutionnelles anti-Noirs. Plus important encore, le travail de Cedillo dans le cadre de la BlackCrit révèle que même les lieux jugés « ouverts », qui permettent aux étudiants différents modes d'acquisition des savoirs, et plus précisément l'enquête ouverte, ne sont pas à l'abri de manifestations qui dénotent une attitude anti-noire. L'auteure promeut un changement dans le discours passant de l'accessibilité à une remise en question du rôle central que jouent les STEM dans la (re)production du racisme individuel et systémique.

Un commentaire critique par Annette et Noel Gough sert de conclusion à ce numéro. D'abord, le commentaire fait des liens entre le numéro spécial et certaines recherches ultérieures pertinentes (par exemple des études scientifiques critiques). Ensuite, il rassure les lecteurs sur le fait que l'ethos de ce numéro n'a rien de particulièrement nouveau, et dépeint donc une préoccupation multigénérationnelle en matière de théorie et de méthode/méthodologie qui persiste en recherche didactique (y compris dans les disciplines des STEM). Enfin, le texte suggère plusieurs avenues à explorer pour les enseignants et les chercheurs intéressés.

Sans plus tarder, nous invitons les lecteurs à prendre le temps de réfléchir sur les sujets, les idées et les outils ayant des liens potentiels avec la recherche et la pratique, et ainsi, peut-être, à faire un premier pas vers des façons de faire différentes. Nous pouvons affirmer que le fait d'être exposé à la « diversité perturbante » de ce numéro spécial a provoqué en nous de nombreux petits changements au cours des 18 derniers mois, et nous espérons que l'esprit du numéro perdurera de multiples façons, pour le bien des écologies de rapports dans lesquels nous vivons, les humains, les non humains et les « plus-qu'humains » avec lesquels nous coexistons. Enfin, nous sommes d'accord avec Gough et Gough pour dire que ce travail n'est ni nouveau, ni près d'être complété. Pour que ce travail puisse refuser, contrer et redéfinir le signifié des champs d'études en STEM, pour qu'il soit exemplaire au lieu de lui être étranger, il est essentiel qu'on y trouve une pluralité, une masse critique, ainsi que des manifestations créatives de perception et de pratiques: encore une fois, l'enseignement des STEM a besoin de perturbation et de déplacement méthodologiques pour qu'il y ait même une possibilité de justice à venir.

\section{Références}

Ahenakew, C. (2016). Grafting Indigenous ways of knowing onto non-Indigenous ways of being. International Review of Qualitative Research, 9(3), 323-340.

Bang, M., \& Marin, A. (2015). Nature-culture constructs in science learning: Human/non-human agency and intentionality. Journal of Research in Science Teaching, 52(4), 530-544.

Derrida, J. (1976). Of grammatology. Trans. G. C. Spivak. Baltimore, MD: John Hopkins University Press.

Gough, N. (2006). Shaking the tree, making a rhizome: Towards a nomadic geophilosophy of science education. Educational Philosophy and Theory, 38(5), 625-645.

Higgins, M., Madden, B., Bérard, M.-F., Lenz Kothe, E., \& Nordstrom, S. (2017). De/signing research in education: Patchwork(ing) methodologies with theory. Educational Studies, 43(1), 16-39.

Jackson, A. Y., \& Mazzei, L. A. (2012). Thinking with theory in qualitative research: Viewing data across multiple perspectives. New York, NY: Routledge.

Jackson, A. Y., \& Mazzei, L. A. (2017). Thinking with theory: A new analytic for qualitative inquiry. In N. K. Denzin, Y. S. Lincoln (Eds.), The Sage Handbook of Qualitative Research (5th ed., pp. 717-727). Thousand Oaks, CA: Sage.

Lather, P. (2007). Getting lost: Feminist practices toward a double(d) science. Albany: SUNY.

McKinley, E. (2001). Cultural diversity: Masking power with innocence. Science Education, 85(1), 74-76.

Smith, L. T., Maxwell, T. K., Puke, H., \& Temara, P. (2016). Indigenous knowledge, methodology and mayhem: What is the role of methodology in producing Indigenous insights? A discussion from mātauranga Māori. Knowledge Cultures, 4(3), $131-156$.

Spivak, G. C. (1976). Translator's preface. In J. Derrida, Of grammatology (G. C. Spivak, Trans.) (pp. ix-lxxxvii). Baltimore, MD: Johns Hopkins University Press. 